\title{
Optimization of an innovative approach involving mechanical activation and acid digestion for the extraction of lithium from lepidolite
}

\author{
Nathália Vieceli ${ }^{1)}$, Carlos A. Nogueira ${ }^{2)}$, Manuel F. C. Pereira ${ }^{3)}$, Fernando O. Durão $^{3)}$, \\ Carlos Guimarães ${ }^{3)}$, and Fernanda Margarido ${ }^{1)}$ \\ 1) Center for Innovation, Technology and Policy Research - IN+, Instituto Superior Técnico, University of Lisbon, 1049-001 Lisboa, Portugal \\ 2) LNEG - Laboratório Nacional de Energia e Geologia, I.P., Campus do Lumiar, 1649-038 Lisboa, Portugal \\ 3) CERENA - Centro de Recursos Naturais e Ambiente, DECivil, Instituto Superior Técnico, University of Lisbon, 1049-001 Lisboa, Portugal \\ (Received: 8 January 2017; revised: 25 August 2017; accepted: 28 August 2017)
}

\begin{abstract}
The recovery of lithium from hard rock minerals has received increased attention given the high demand for this element. Therefore, this study optimized an innovative process, which does not require a high-temperature calcination step, for lithium extraction from lepidolite. Mechanical activation and acid digestion were suggested as crucial process parameters, and experimental design and response-surface methodology were applied to model and optimize the proposed lithium extraction process. The promoting effect of amorphization and the formation of lithium sulfate hydrate on lithium extraction yield were assessed. Several factor combinations led to extraction yields that exceeded $90 \%$, indicating that the proposed process is an effective approach for lithium recovery.
\end{abstract}

Keywords: lepidolite; lithium; mechanical activation; acid digestion; optimization; extraction

\section{Introduction}

Strategic and critical metals are of great importance given their application in emerging technologies. New metal sources must be identified and existing extraction technologies must be optimized to ensure the continued supply of these metals to a growing market. Although lithium is not classified as a critical element in some lists (such as in the European Union), it is a strategic metal due to its relevance in energy and mobility. Specifically, lithium is used as a raw material for batteries for plug-in hybrid and electric vehicles, as well as for electrochemical energy storage.

Natural lithium resources include brines and pegmatite minerals. For many years, brine exploitation has been sufficient to fulfill the global lithium demand. Lithium recovery from hard rock minerals, however, has attracted interest again, and several studies on this topic have been reported. In these studies, a salt-roasting step is used as an important alternative to the traditional process of calcination. The salt-roasting step is then followed by acid digestion. The uti- lization of additives has decreased calcination time and temperature, although calcination temperatures still range from 800 to $1000^{\circ} \mathrm{C}$ [1-15]. The majority of these studies involve the mineral lepidolite, an important lithium resource that has recently gained attention as an alternative to spodumene, the most common mineral source of lithium.

Despite the scientific advances mentioned above, the proposed processes always involve an economically and environmentally unfavorable high-temperature step. Therefore, this study applied an alternative process that involves mechanical activation followed by acid digestion and water leaching to extract lithium from lepidolite. Vieceli et al. [16] have previously discussed the effects and associated transformations of this process. This process is a noteworthy approach to lithium extraction from lepidolite concentrate because it allows the replacement of high-temperature calcination. The present study aimed to evaluate and optimize the factors that affect the digestion of an activated lepidolite concentrate. Factorial design and response surface methodologies were utilized to achieve this objective. 